\title{
EQ-5D-Y Population Norms for Japanese Children and Adolescents
}

\author{
Takeru Shiroiwa $^{1}$ (D) $\cdot$ Takashi Fukuda $^{1}$
}

Accepted: 27 June 2021 / Published online: 22 July 2021

(c) The Author(s) 2021

\begin{abstract}
Objective This study was aimed at establishing population norms of EQ-5D-Y in Japan by a nationwide large sample survey. Methods We performed a door-to-door survey by visiting the homes of children/adolescents aged 8-15 years selected by random sampling. The planned sample size was 3600 from 100 districts in Japan. Children/adolescents were asked to respond to the EQ-5D-Y instrument, and their parents, to background questions on themselves and their children. Summary statistics by age/sex were calculated to obtain the Japanese population norms. A multiple linear regression model was used to examine the relationships between the EQ-5D-Y index and their parents' demographic factors, the children/adolescents' diseases/symptoms and the family environment.

Results We collected 3636 responses from 100 districts. The overall EQ-5D-Y index values (all sexes, ages) ranged from 0.90 to 0.95 . The percentage of respondents reporting full health ranged from 40 to $60 \%$. In regard to the influence of the children/adolescents' diseases/symptoms on disutility, developmental disability showed the largest disutility values of 0.090 . Sleeplessness and body pain were the symptoms that exerted the greatest influence on the EQ-5D-Y index; the effect sizes ranged from -0.030 to -0.098 for sleeplessness, and from -0.023 to -0.079 for body pain. The EQ-5D-Y index of children/adolescents with parents who reported severe stress was lower by $0.072(p<0.001)$, as compared with that of children/ adolescents with parents reporting no stress.

Conclusions Population norms of EQ-5D-Y were established for the first time. We also clarified the relationship between the EQ-5D-Y index value and the children/adolescents' diseases/symptoms and the family environment.
\end{abstract}

\section{Key Points}

We established population norms of EQ-5D-Y in Japan. The mean EQ-5D-Y index value did not depend on the age or sex

We constructed a disutility catalogue of diseases/symptoms of the children/adolescents. Developmental disability were associated with large disutility scores, as were sleeplessness and body pain

Stress in the parents and some lifestyles of the children and adolescents were associated with significantly decreased EQ-5D-Y index values

Takeru Shiroiwa

t.shiroiwa@gmail.com

1 Center for Outcomes Research and Economic Evaluation for Health $(\mathrm{C} 2 \mathrm{H})$, National Institute of Public Health, 2-3-6 Minami, Wako, Saitama 351-0197, Japan

\section{Introduction}

A number of preference-based measures (PBMs) have been developed for children/adolescents, such as the Child Health Utility 9 dimensions (CHU-9D) [1], EQ-5D-Y [2], Health Utilities Index (HUI) [3], 16D [4] or 17D [5], and The Assessment of Quality of Life (AQoL-6D) [6]. The EQ-5D-Y is a PBM that has been developed to measure the health states of children/adolescents (aged 8-15 years). It is a child-friendly version of and conceptually equivalent to the adult version of EQ-5D. The description of each item and the severity levels are adapted so as to make them more relevant to younger populations. After the new instrument is established, a value set is needed to convert the responses to the EQ-5D-Y to index values, which are anchored to 0 (death) or 1 (full health). Value sets of EQ$5 \mathrm{D}-\mathrm{Y}$ have already been constructed in some countries, including Japan and Slovenia [7, 8], by valuation surveys based on an international common protocol [9]. The calculated index values can be used for cost-effectiveness 
analyses to estimate the quality-adjusted life-years by weighting obtained life-years.

To interpret the collected responses to PBMs, population norms are widely used in every country. Population norms are defined as "population reference data ... for a specific country or international region" [10]. Population norms can be used as a reference for comparison with the obtained data. In addition, population norms can also be applied to cost-effectiveness analyses, by considering them as the values in people without any diseases or symptoms. Population norms of some PBMs have already been established in some countries. For example, the population norms of EQ-5D-5L have been reported for various countries or regions [11-26], including Japan [27, 28], although some reports only provide the distribution of the EQ-5D-5L responses without the index values. In contrast, population norms of PBMs for children/adolescents, including of the EQ-5D-Y, have not yet been established in any country. It is highly likely that the population norms of children/adolescents are different from those of adults.

The primary objective of this study was to establish the population norms of EQ-5D-Y, based on a nationwide random sample survey in Japan. The norms are presented by age and sex. A second objective was to investigate the relationships between the EQ-5D-Y index values and diseases and symptoms in children/adolescents. The estimated coefficients can also be applied to cost-effectiveness analyses as disutility values of the patients with diseases or symptoms. A third objective was to explore the influence of the family environment (mental health of the parents and lifestyles of the children and adolescents) on the EQ-5D-Y index values. For children/adolescents, the family environment, including the parents' life situation, may exert an influence on their health states; we explored the quantitative relationships between the EQ-5D-Y index values and the family environment to understand this influence. Through these objectives, we aim to promote a better understanding of the health states and quality of life of children/ adolescents.

\section{Methods}

\subsection{Instruments}

The EQ-5D-Y(-3L) is composed of five dimensions, each assessed at three levels: "mobility", "looking after myself", "doing usual activities", "having pain or discomfort", and "feeling worried, sad, or unhappy". It covers the same basic dimensions of health as the adult version, EQ-5D, but the words and phrases are simplified to make the instrument more child friendly; it is thus slightly different from EQ-5D-3L or 5L $[29,30]$. For example, the fifth item is changed from anxiety/depression into "feeling worried, sad, or unhappy". EQ-5D-Y is designed for self-reporting by children/adolescents aged 8-15 years. It might also be possible to use the adult EQ-5D version for children/adolescents aged $12-15$ years, although only the adult EQ-5D would be applicable to adolescents aged 16 years or over [31].

We also included in our survey some questions about diseases and symptoms that the children and adolescents might be experiencing. They were asked to select one of three categories ("none", "occasionally", and "frequently") that best fitted their condition for the case of symptoms, and one of two categories ("no" and "yes") for the case of diseases. Items on the demographic characteristics of the parents and family environment (stress level in the parents, degree of happiness of the parents, and lifestyle of the children and adolescents) were also added to the questionnaire. Although the children/adolescents themselves requested to provide the responses to the items on the EQ-5D-Y, the parents responded to the other questions.

\subsection{Respondents}

The respondents were children/adolescents aged $8-15$ years and their parents. They were randomly sampled from 100 districts throughout Japan. The planned sample size was 3600, with an expected 225 subjects in every age and sex category. A door-to-door survey was conducted from January 2019 to March 2019. Investigators visited their addresses registered with the respective municipalities and distributed the questionnaire forms. Children/adolescents and their parents were asked to self-complete the instruments and respond to the questions. Children/adolescents who were hospitalized or admitted in orphanages were excluded. If the investigators obtained informed consent for participation, they returned to collect the forms after a few days and checked for missing data. If some responses were missing, the investigators asked them to respond to the missing items. These visits by the investigators were continued until the planned number of responses were collected from each district.

\subsection{Sampling Method}

The 100 districts (Cho-me, in Japanese) were selected as follows. First, the number of surveyed districts in each of the eight regions of Japan (Hokkaido, Tohoku, Kanto, Chubu, Kinki, Chugoku, Shikoku, and Kyushu) was determined in proportion to the population in each region. Then, for each region, the number of municipalities belonging to each stratum (i.e., prefectures in each region $\times$ three size categories [large cities, medium-sized cities, and small towns] of municipality) was calculated based on the population of the stratum. Surveyed municipalities were randomly selected according to the allocated number in each stratum. Next, 
the surveyed districts, which are located in the selected municipality, were randomly selected from all the districts in each municipality. Finally, respondents were also randomly sampled from each selected district, and stratified by sex and age. Individuals admitted to hospitals and nursing homes were excluded. Respondents were randomly sampled from the list of The Basic Resident Register. In Japan, each municipality has its own Basic Resident Register data, which include information such as the name, sex, address, and date of birth of individuals. We obtained permission from each municipality to use the data for our study.

\subsection{Statistical Analysis}

Responses to the EQ-5D-Y were converted into index values using the Japanese value sets [7]. Summary index values by age and sex were calculated to obtain the normative values for Japanese children/adolescents. The percentages of individuals who reported no problems with responding to the EQ-5D-Y were also computed.

A multiple linear regression model was used to examine the relationships between the EQ-5D-Y index values and demographic factors. Age, sex, household income, employment status, education level, and marital status were included in the model. Next, we used three different multiple linear models. First, to estimate the size of the disutility associated with diseases, a linear regression model including the age category, sex, and disease was constructed to estimate the regression coefficients. Second, considering the effect of symptoms, a similar linear model was constructed, replacing diseases with symptoms. Finally, to consider the effects of the family environment of the children/adolescents (level of stress in the parents, degree of happiness of the parents, bedtime of the children/adolescents, and frequency at which they ate breakfast alone), a linear regression model including the age category, sex, and each factor related to every family environment was constructed. Using a robust standard error, the $p$ value was calculated.

All the statistical analyses were performed using SAS 9.4. This study was conducted with the approval of the Ethics Committee of the National Institute of Public Health (NIPHIBRA \#12179).

\section{Results}

\subsection{Response Rate and Demographic Factors}

We collected 3636 responses from the 100 selected districts. The overall response rate was $45.2 \%$, and the response rates by region were as follows; $43.8 \%$ from
Hokkaido, 51.1\% from Tohoku, $42.6 \%$ from Kanto, 49.6\% from Chubu, $43.8 \%$ from Kinki, $51.0 \%$ from Chugoku, 51.8\% from Shikoku, and 45.3\% from Kyushu. The response rates did not largely differ among the regions. When the municipality in which the respondents lived were classified into three categories by the population (large cities, medium-sized cities, and small towns), the response rate was $43.6 \%$ from large cities, $45.8 \%$ from mediumsized cities, and $48.4 \%$ from small towns. Thus, the size of the city in which the respondents resided appeared to be related to the response rate. The demographic characteristics of the respondent children/adolescents and their parents are shown in Table 1. The population in each region as of October 2019, as estimated by the Japanese government, was $4.2 \%$ (Hokkaido), $6.9 \%$ (Tohoku), 34.4\% (Kanto), 16.8\% (Chubu), 17.7\% (Kinki), 5.8\% (Chugoku), $2.9 \%$ (Shikoku), and $11.3 \%$ (Kyushu). Our sampling did not involve a regional bias. The average household income of the whole population with children in 2018 was JPY 7.5 million (USD 69,000, 1 USD = $108 \mathrm{JPY}$, as of March 2021 ), with a median income of JPY 6.0 million (USD 56,000 ). Our data showed that the median category of household income was JPY 6.0 million to JPY 10 million per year, which was comparable to that of the overall Japanese population with children. In regard to other factors, as it was difficult to find Japanese government statistics on families with children and/or adolescents, no comparable data to those shown in Table 1 exist.

\subsection{Population Norms of EQ-5D-Y}

Population norms of EQ-5D-Y, the summary statistics of index values, are shown in Table 2 and Fig. 1, stratified by the age and sex of the subjects. Table 2 also shows the percentage of respondents who reported being in a full health state by EQ-5D-Y. The mean EQ-5D-Y index values did not depend on the age or sex. These values ranged from 0.90 to 0.95 for all ages and both sexes. The percentage of respondents in full health ranged from 40 to $60 \%$. Conversely, about a half of the children/adolescents reported at least one health problem. However, as even the 25th percentile EQ-5D-Y values were $0.88-0.93$, the health problems did not seem severe.

Responses to each item are also shown in Table 3. The item for which most respondents reported not being in a full health state were "feeling worried, sad, or unhappy". About only $65 \%$ of children and adolescents reported having no problem in relation to this item. In contrast, more than $90 \%$ of respondents reported being in a full health state for the "looking after myself" item. 
Table 1 Demographic characteristics of the respondents

\begin{tabular}{|c|c|c|}
\hline & $N$ & Percentage \\
\hline \multicolumn{3}{|l|}{ Age, years } \\
\hline 8 & 455 & 12.5 \\
\hline 9 & 453 & 12.5 \\
\hline 10 & 453 & 12.5 \\
\hline 11 & 461 & 12.7 \\
\hline 12 & 451 & 12.4 \\
\hline 13 & 457 & 12.6 \\
\hline 14 & 450 & 12.4 \\
\hline 15 & 454 & 12.5 \\
\hline \multicolumn{3}{|l|}{ Sex } \\
\hline Male & 1817 & 50.0 \\
\hline Female & 1817 & 50.0 \\
\hline \multicolumn{3}{|l|}{ Region } \\
\hline Hokkaido & 145 & 4.0 \\
\hline Tohoku & 259 & 7.1 \\
\hline Kanto & 1267 & 34.9 \\
\hline Chubu & 545 & 15.0 \\
\hline Kinki & 645 & 18.0 \\
\hline Chugoku & 219 & 6.0 \\
\hline Shikoku & 109 & 3.0 \\
\hline Kyushu & 436 & 12.0 \\
\hline \multicolumn{3}{|c|}{ Household income (JPY 1 million) } \\
\hline$<1$ & 27 & 0.7 \\
\hline $1<=<2$ & 78 & 2.1 \\
\hline $2<=<4$ & 395 & 10.9 \\
\hline $4<=<6$ & 925 & 25.5 \\
\hline $6<=<10$ & 1544 & 42.5 \\
\hline $10<=<15$ & 484 & 13.3 \\
\hline $15<=<20$ & 91 & 2.5 \\
\hline $20<=$ & 35 & 1.0 \\
\hline Missing & 55 & 1.5 \\
\hline \multicolumn{3}{|l|}{ Parents' marital status } \\
\hline Married & 3344 & 92.0 \\
\hline Others (unmarried, divorced) & 290 & 8.0 \\
\hline \multicolumn{3}{|l|}{ Parents' employment status } \\
\hline Both parents are working & 2850 & 78.4 \\
\hline One of the parents is working & 755 & 20.8 \\
\hline Neither parent is working & 29 & 0.8 \\
\hline \multicolumn{3}{|c|}{ Parents' history of education (university or graduate) } \\
\hline Both parents & 609 & 16.8 \\
\hline Either parent & 1017 & 28.0 \\
\hline Neither & 1706 & 46.9 \\
\hline Missing & 302 & 8.3 \\
\hline
\end{tabular}

\subsection{Relationships Between the EQ-5D-Y Index Values and Demographic Factors}

The results of the analysis using the model that included the parents' demographic factors are shown in Table 4. The marital status, education history, and working status exerted no influence on the EQ-5D-Y index values of the children/ adolescents; in contrast, household income was associated with the values. That is, the higher the household income, the higher the EQ-5D-Y index value, although the effect size was small (0.002 per JPY 1 million). In the case of social security payments, the EQ-5D-Y values of those who were receiving social security payments were lower by 0.023 as compared with those who were not receiving social security payments. The values of those who had previously received, but were no longer receiving social security payments were lower by 0.013 . Neither of the aforementioned differences was statistically significant $(p=0.376$ and $p=$ 0.631 , respectively); note that the number of respondents was limited ( $n=12$ and $n=13$, respectively).

\subsection{Disutility Associated with Diseases and Symptoms in the Children/Adolescents}

Table 5 shows the relationships between the EQ-5D-Y values and parent-reported diseases of the children/adolescents. No pediatric diseases other than heart disease, asthma, ear diseases, epilepsy, and influenza exerted a significant influence on the EQ-5D-Y values. Among other diseases (atopic dermatitis, allergic rhinitis, developmental disability, and cold/gastroenteritis), developmental disability was associated with the largest disutility values of 0.090 . Cold/gastroenteritis exerted a moderate influence on the EQ-5D-Y values (the disutility values were 0.040 ).

Table 6 shows the relationships between the EQ-5D-Y values and the parent-reported symptoms in the children/ adolescents. The responses for the frequency of symptoms were classified into three categories (none, occasionally, frequently), and the estimated coefficients did not show any inconsistency, i.e., no case of "frequently" showed a disutility value lower than the values for "occasionally" or "none". The symptoms that exerted the greatest influence on the EQ-5D-Y values were sleeplessness and body pain. The sizes for sleeplessness were -0.030 for "occasionally" and -0.098 for "frequently", and those for body pain were -0.023 for "occasionally" and -0.079 for "frequently".

\subsection{Disutility Associated with the Family Environment of the Children/Adolescents}

The EQ-5D-Y index was significantly associated with the degree of stress in the parents (Appendix). Self-reported parents' stress was classified into four categories (very much, somewhat, hardly, none). The EQ-5D-Y values of children/adolescents whose parents reported being very much stressed $(n=295)$ was lower by $0.072(p<0.001)$, and that 
Table 2 Population norms of EQ-5D-Y

\begin{tabular}{|c|c|c|c|c|c|c|c|c|}
\hline \multirow[t]{2}{*}{ Age, years } & \multirow[t]{2}{*}{ Sex } & \multicolumn{7}{|c|}{ EQ-5D-Y index } \\
\hline & & $N$ & Mean & SD & Q1 & Median & Q3 & $\%$ of full health \\
\hline \multirow[t]{2}{*}{8} & Male & 226 & 0.927 & 0.088 & 0.888 & 0.937 & 1.000 & 42.9 \\
\hline & Female & 229 & 0.935 & 0.078 & 0.898 & 0.937 & 1.000 & 42.8 \\
\hline \multirow[t]{2}{*}{9} & Male & 266 & 0.922 & 0.084 & 0.880 & 0.926 & 1.000 & 39.9 \\
\hline & Female & 187 & 0.925 & 0.087 & 0.886 & 0.926 & 1.000 & 40.6 \\
\hline \multirow[t]{2}{*}{10} & Male & 232 & 0.927 & 0.093 & 0.887 & 0.935 & 1.000 & 43.5 \\
\hline & Female & 221 & 0.942 & 0.086 & 0.926 & 1.000 & 1.000 & 53.4 \\
\hline \multirow[t]{2}{*}{11} & Male & 220 & 0.930 & 0.092 & 0.888 & 0.937 & 1.000 & 48.2 \\
\hline & Female & 241 & 0.934 & 0.093 & 0.897 & 1.000 & 1.000 & 50.6 \\
\hline \multirow[t]{2}{*}{12} & Male & 216 & 0.931 & 0.094 & 0.898 & 0.937 & 1.000 & 48.6 \\
\hline & Female & 235 & 0.931 & 0.097 & 0.898 & 0.937 & 1.000 & 49.8 \\
\hline \multirow[t]{2}{*}{13} & Male & 222 & 0.934 & 0.083 & 0.888 & 1.000 & 1.000 & 51.4 \\
\hline & Female & 235 & 0.911 & 0.113 & 0.858 & 0.926 & 1.000 & 42.1 \\
\hline \multirow[t]{2}{*}{14} & Male & 199 & 0.927 & 0.097 & 0.888 & 0.937 & 1.000 & 48.2 \\
\hline & Female & 251 & 0.930 & 0.099 & 0.886 & 1.000 & 1.000 & 51.8 \\
\hline \multirow[t]{2}{*}{15} & Male & 236 & 0.938 & 0.114 & 0.926 & 1.000 & 1.000 & 58.5 \\
\hline & Female & 218 & 0.931 & 0.100 & 0.898 & 0.936 & 1.000 & 48.2 \\
\hline
\end{tabular}

$S D$ standard deviation

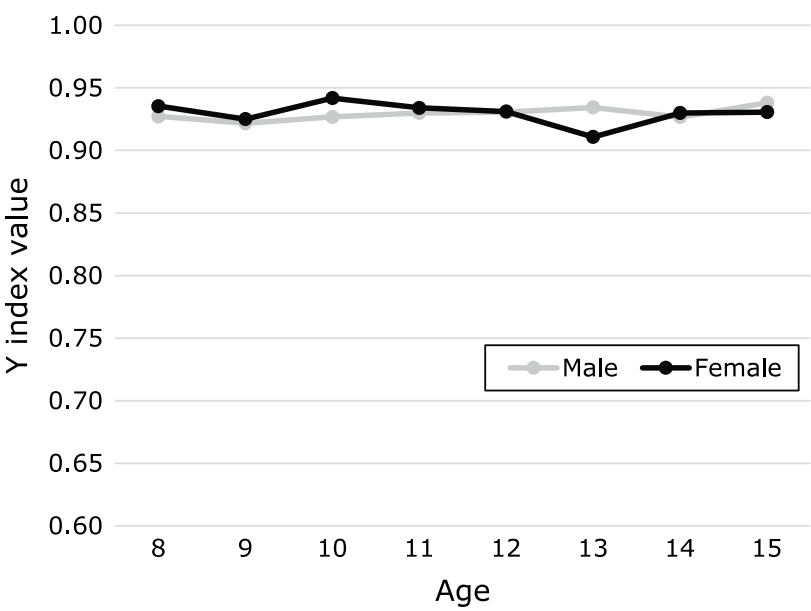

Fig. 1 Population norms by sex and age

Table 3 Distribution of responses

\begin{tabular}{llll}
\hline Item & \multicolumn{4}{l}{ Response, $n(\%)$} \\
\cline { 2 - 4 } & 1 & 2 & 3 \\
\hline Mobility & $3080(84.8)$ & $494(13.6)$ & $60(1.7)$ \\
Looking after myself & $3328(91.6)$ & $279(7.7)$ & $27(0.7)$ \\
Doing usual activities & $3035(83.5)$ & $541(14.9)$ & $58(1.6)$ \\
Having pain or discomfort & $2834(78.0)$ & $747(20.6)$ & $53(1.5)$ \\
$\begin{array}{l}\text { Feeling worried, sad, or } \\
\quad \text { unhappy }\end{array}$ & $2356(64.8)$ & $1135(31.2)$ & $143(3.9)$ \\
& & & \\
\end{tabular}

Table 4 Relationships between the EQ-5D-Y index values and demographic factors

\begin{tabular}{lcll}
\hline & Estimate & Standard error & $p$ value \\
\hline Intercept & 0.961 & 0.026 & $<0.001$ \\
Children's age & 0.000 & 0.000 & 0.629 \\
Children's sex & 0.002 & 0.003 & 0.620 \\
Household income (JPY1 mil- & 0.002 & 0.000 & $<0.001$ \\
$\quad$ lion) & & & \\
Parents' marital status & - & - & - \\
Married & 0.032 & 0.020 & 0.113 \\
Others (unmarried, divorced) & & & \\
Parents' employment status & - & - & - \\
Both parents are working & -0.010 & 0.015 & 0.478 \\
One of the parents is working & -0.008 & 0.015 & 0.211 \\
Neither parent is working & - & - & \\
Parents' history of education (university or & graduate) & \\
Both parents & - & 0.005 & 0.257 \\
Either parent & 0.005 & 0.006 & 0.817 \\
Neither & \multicolumn{2}{c}{0.004} & \\
\hline
\end{tabular}

of children/adolescents whose parents reported being somewhat stressed $(n=2437)$ was lower by $0.022(p=0.001)$ as compared with that of the children/adolescents whose parents reported no stress (none). In addition, the happiness level of the parents also significantly influenced the EQ5D-Y values of the children/adolescents. The EQ-5D-Y values of children/adolescents whose parents' happiness level 
Table 5 Disutility scores associated with diseases

\begin{tabular}{|c|c|c|c|c|}
\hline & $N$ & Estimate & Standard error & $p$ value \\
\hline Intercept & & 0.942 & 0.008 & $<0.001$ \\
\hline Age & & -0.000 & 0.000 & 0.790 \\
\hline Sex & & 0.001 & 0.003 & 0.634 \\
\hline
\end{tabular}

Disease

Heart disease

No 3604

Yes

Asthma

$\begin{array}{lll}30 & -0.036 & 0.026\end{array}$

$-$

No

$3470-$

Yes

$164-0.011$

$-$

Atopic dermatitis

No 3366

$\begin{array}{llll}\text { Yes } & 268 & -0.013 & 0.007\end{array}$

Ear disease (otitis media)

No 3592 -

Yes

420.005

.005

$-$

Allergic rhinitis

No

Yes

Epilepsy

No

Yes

Developmental disability

No

Yes

Influenza

No

Yes

$\begin{array}{rll}2811 & - & - \\ 823 & -0.011 & 0.00\end{array}$

0.017

0.004

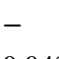

0.043

Cold or gastroenteritis

\begin{tabular}{lrlll} 
No & 3505 & - & - & - \\
Yes & 129 & -0.040 & 0.012 & 0.001 \\
Tooth decay & & & & \\
No & 3276 & - & - & - \\
Yes & 358 & 0.023 & 0.006 & $<0.001$ \\
\hline
\end{tabular}

was 6 or lower on a scale of 10 were lower. The disutility size was 0.045 ( $n=42, p=0.026)$ for a happiness level of $1-3$ on a scale of 10 and $0.036(n=457, p<0.001)$ for a happiness level of 4-6 on a scale of 10 , as compared to a happiness level of 7-10 on a scale of 10. It is possible that the parents' mental health condition influences the health states of their children/adolescents, although the causal relation remains unclear.

In addition, in regard to the lifestyles of the children/ adolescents, their bedtime was significantly associated with the EQ-5D-Y values. Children/adolescents who went to bed before 10 p.m. ( $n=1275$ ) had higher EQ-5D-Y values as compared with children/adolescents who went to bed between 11 p.m. and midnight $(-0.021, p<0.001, n=$
Table 6 Disutility associated with symptoms

\begin{tabular}{|c|c|c|c|c|}
\hline & $N$ & Estimate & Standard error & $p$ value \\
\hline Intercept & & 0.925 & 0.009 & $<0.001$ \\
\hline Age & & 0.002 & 0.000 & 0.013 \\
\hline Sex & & 0.007 & 0.003 & 0.013 \\
\hline \multicolumn{5}{|l|}{ Symptoms } \\
\hline \multicolumn{5}{|l|}{ Headache } \\
\hline No & 2605 & - & & - \\
\hline Occasionally & 913 & -0.011 & 0.004 & 0.004 \\
\hline Frequently & 116 & -0.027 & 0.011 & 0.011 \\
\hline \multicolumn{5}{|l|}{ Stomachache } \\
\hline No & 2396 & - & & - \\
\hline Occasionally & 1103 & -0.009 & 0.003 & 0.016 \\
\hline Frequently & 135 & -0.023 & 0.012 & 0.058 \\
\hline \multicolumn{5}{|l|}{ Nausea } \\
\hline No & 3218 & - & & - \\
\hline Occasionally & 390 & -0.031 & 0.006 & $<0.001$ \\
\hline Frequently & 26 & -0.046 & 0.020 & 0.020 \\
\hline \multicolumn{5}{|l|}{ Sleeplessness } \\
\hline No & 3359 & - & & - \\
\hline Occasionally & 238 & -0.030 & 0.006 & $<0.001$ \\
\hline Frequently & 37 & -0.098 & 0.038 & $<0.001$ \\
\hline \multicolumn{5}{|c|}{ Body pain (shoulder, back, and neck) } \\
\hline No & 2663 & - & & - \\
\hline Occasionally & 856 & -0.023 & 0.004 & $<0.001$ \\
\hline Frequently & 115 & -0.079 & 0.013 & $<0.001$ \\
\hline \multicolumn{5}{|l|}{ Appetite loss } \\
\hline No & 3395 & - & & - \\
\hline Occasionally & 221 & -0.028 & 0.008 & $<0.001$ \\
\hline Frequently & 18 & -0.102 & 0.057 & 0.074 \\
\hline
\end{tabular}

807), between midnight and 1 a.m. $(-0.028, p<0.001$, $n=282)$, or at 1 a.m. or later $(-0.080, p<0.001, n=$ 59). Finally, the EQ-5D-Y values of children/adolescents who had breakfast with their family members everyday ( $n$ $=3317$ ) were significantly higher than those of children/ adolescents who had breakfast alone everyday $(n=75)$; the disutility size was $0.057(p=0.003)$.

\section{Discussion}

This large sample survey conducted by random sampling yielded the following. First, population norms in Japan were established, and this is the first report of establishment of the population norms of EQ-5D-Y. The EQ-5D-Y values did not differ depending on the age or sex of the children/ adolescents. Second, we obtained the relationships between the EQ-5D-Y values of the children/adolescents and the demographic characteristics of their parents. Only household income was identified as a statistically significant factor, but 
the effect size was small. Third, we constructed a disutility catalogue of the children/adolescents' diseases and symptoms. Developmental disability were associated with large disutility values, as were the symptoms of sleeplessness and body pain. Finally, some mental states of the parents and some lifestyles of the children/adolescents were associated with significantly decreased EQ-5D-Y values. Our results also show that surveys based on the EQ-5D-Y are feasible. The results of the assessment of the EQ-5D-Y were sufficiently interpretable, and clear dose-response relationships were detected.

According to the population norms of EQ-5D-5L in Japan [28], the mean value for respondents aged 16-19 years is 0.978 for male individuals and 0.967 for female individuals. More than $80 \%$ were found to be in a full health state $(85.9 \%$ [male] and $80.6 \%$ [female]). The index value of the respondents aged 60-69 years was 0.930 for male individuals and 0.927 for female individuals; $60.9 \%$ of the male respondents and $55.7 \%$ of all the respondents were found to be in a full health state. In comparison, according to the EQ-5D-Y norms, the percentage of children/adolescents in a full health state was smaller. It shows similar percentage with respondents aged 60-69 years. As it is highly likely that the actual average health states are not different between respondents aged 15 years or under and aged $16-19$ years, it is possible that the responsiveness of the two Japanese EQ-5D instruments are different.

The Japanese EQ-5D-Y value for the worst health state [33333] was 0.29, which was much higher than the adult EQ-5D value (- 0.11 in EQ-5D-3L [32] and - 0.03 in EQ$5 \mathrm{D}-5 \mathrm{~L}$ [33]). The disutility values according to the Japanese EQ-5D-Y scoring algorithm tend to be smaller than those estimated by adult EQ-5D instruments for each item and level. Therefore, it may be unreasonable to conduct a simple comparison of our estimated EQ-5D-Y disutility values with those measured by adult EQ-5D instruments. For example, it is likely that a disutility value of 0.1 estimated by EQ-5D-Y does not correspond to a similar degree of deterioration of the health states as a disutility value of 0.1 measured by the adult EQ-5D instrument. Although the minimal important difference of EQ-5D-Y has not been reported yet, it could be smaller than that of adult EQ-5D [34]. These differences should be taken into consideration while interpreting our results; problems may arise if these differences are ignored. For example, it is good for children that health technologies to prolong children's or adolescents' life-years have become more cost effective than those for adults. However, assuming that a healthcare technology can restore a patient's health state from a worse state to full health, the improvement in the children/adolescents' quality of life would be smaller than that of adults.
When the Japanese EQ-5D-5L population norms were analyzed, sex, household income, and length of education were found to be significantly associated with the index values. [28] However according to our survey, the sex of the children/adolescents had no influence on the index values, nor had the parents' characteristics, except for household income, the effect size of which was also not large. The respondents' own demographic characteristics and their parents' demographic characteristics may exert different influences on the health states and/or disutility. In the case of the Japanese EQ-5D-5L population norms, Parkinson's disease (0.35), stroke (0.27), dementia (0.22), and depression (0.18) are associated with the largest disutility values. However, in the EQ-5D-Y, developmental disability were associated with the largest disutility values of 0.090 . The disutility associated with disease according to the EQ-5D-Y seems smaller, but it is not yet clear if that is dependent on the type of disease or the characteristics of the instrument.

The sample size in our survey was 3600 . In this area, the sample size cannot be determined by statistical considerations, but the sample size is important for the representativeness and accuracy of the population norms. One of the largest surveys, as compared to the EQ-5D-5L population norms survey, was performed in Spain [14]. The sample size was more than 20,000. However, a German survey was conducted on 5000 subjects [21], a Polish survey on about 4000 subjects [15], and an Australian and Quebec survey on about 3000 subjects [13, 24]. About 1000 respondents were sampled for establishing the norms in Hong Kong [22], USA (only face-to-face survey) [26], Japan [27], Ireland [19], Bulgaria [25], Slovenia [23], and Indonesia [17]. Considering these existing studies, our sample size was comparable and sufficiently large to estimate population norms.

We believe that the population norms of EQ-5D-Y established by us are scientifically valid; however, they may present some difficult issues for the decision makers. Our results showed that the Japanese EQ-5D-Y population norms for children/adolescents aged 15 years or under were not consistent with those of the respondents aged 16-19 years as measured by the EQ-5D-5L, despite presumably similar health states. When both the EQ-5D-Y and 5L norms are used for cost-effectiveness analyses of pediatric interventions, it may be difficult to justify applying lower norms only to children/adolescents aged 8-15 years and higher norms for those aged 16-19 years. It is also difficult to explain why the switching of the two (EQ-5D-Y-based and 5L-based) population norms occurred at the age of 16 years, and not at the age of 15 or 17 years, for example; the reasons are not health related. These issues may need to be clarified, especially when our population norms are applied to costeffectiveness analyses for decision making. 
The strength of our study lay in the robust random sampling and the reasonably large sample size. Our sampling method using the Basic Resident Register data was a robust and reliable sampling method. There was no bias related to the area of residence of the sampled respondents. The sample number was relatively high, as mentioned above. Each category stratified by sex and age included more than 200 respondents, which we believe is a sufficient number to obtain reliable results and reasonably interpret the results. In addition, we included a number of background questions, including those related to the parents' demographic characteristics, diseases and symptoms of the subjects, and the family environment. Thus, we were able to explore the relationships between the EQ-5D-Y values and the data obtained about the background factors. In contrast, this study had the following limitation. While our survey was based on a robust random sampling method, the response rate was only about $45 \%$, which may be low. Our survey was a community-based, and we did not include children/ adolescents admitted to hospitals and institutions. Therefore, children/adolescents with severe diseases or in adverse environments were not included in our survey. In addition, in our survey, we requested that the children/adolescents selfcomplete the EQ-5D-Y questionnaire and prohibited proxy responses. However, we could not confirm that it was indeed the children/adolescents who responded to the EQ-5D-Y and their parents to the background questions. Background information, especially in relation to the disease and symptoms, is based on the parents' proxy report. It may be different from the assessment by the clinicians.

\section{Conclusions}

This was the first report of establishment of the EQ-5D-Y population norms for Japan based on a large sample survey. According to the estimated population norms, the EQ-5D-Y values did not differ depending on the age or sex of the children/adolescents. This suggests that it may also be feasible to apply the results of the EQ-5D-Y survey to younger children aged 8 or 9 years. We constructed a disutility catalogue of children/adolescents' diseases and symptoms. Developmental disability associated with large disutility values, as were the symptoms of sleeplessness and body pain. These results can be used for economic evaluation of healthcare technologies for children/adolescents, although the difference in characteristics between EQ-5D-Y and adult EQ-5D would need to be considered.

\section{Appendix}

Relationships between the EQ-5D-Y index values and family environment

\begin{tabular}{|c|c|c|c|c|}
\hline & $N$ & Coefficient & Standard error & $P$ value \\
\hline \multicolumn{5}{|l|}{ (a) Social security payment } \\
\hline Intercept & & 0.927 & 0.009 & $<0.001$ \\
\hline Age & & 0.000 & 0.001 & 0.837 \\
\hline Sex & & 0.001 & 0.003 & 0.850 \\
\hline \multicolumn{5}{|l|}{ Social security payment } \\
\hline Currently & 12 & -0.023 & 0.026 & 0.376 \\
\hline Previously & 13 & -0.013 & 0.028 & 0.631 \\
\hline None & 3609 & - & - & - \\
\hline \multicolumn{5}{|l|}{ (b) Stress } \\
\hline Intercept & & 0.947 & 0.011 & $<0.001$ \\
\hline Age & & 0.000 & 0.001 & 0.732 \\
\hline Sex & & 0.001 & 0.003 & 0.778 \\
\hline \multicolumn{5}{|l|}{ Stress or worries } \\
\hline Very much & 295 & -0.072 & 0.010 & $<0.001$ \\
\hline Somewhat & 2437 & -0.022 & 0.006 & 0.001 \\
\hline Hardly & 773 & -0.007 & 0.007 & 0.282 \\
\hline None & 129 & - & & - \\
\hline \multicolumn{5}{|l|}{ (c) Happiness level } \\
\hline Intercept & & 0.931 & 0.009 & $<0.001$ \\
\hline Age & & 0.000 & 0.001 & 0.647 \\
\hline Sex & & 0.000 & 0.003 & 0.903 \\
\hline \multicolumn{5}{|l|}{ Happiness level } \\
\hline $7-10$ & 3135 & - & & - \\
\hline $4-6$ & 457 & -0.036 & 0.020 & 0.026 \\
\hline $1-3$ & 42 & -0.045 & 0.006 & $<0.001$ \\
\hline \multicolumn{5}{|l|}{ (d) Breakfast } \\
\hline Intercept & & 0.867 & 0.021 & $<0.001$ \\
\hline Age & & 0.001 & 0.001 & 0.292 \\
\hline Sex & & 0.000 & 0.003 & 0.892 \\
\hline \multicolumn{5}{|c|}{ Breakfast with family members } \\
\hline Every day & 3317 & 0.057 & 0.019 & 0.003 \\
\hline 4-6 days per week & 148 & 0.033 & 0.021 & 0.112 \\
\hline $1-3$ days per week & 94 & 0.034 & 0.022 & 0.129 \\
\hline None & 75 & - & & - \\
\hline \multicolumn{5}{|l|}{ (e) Bedtime } \\
\hline Intercept & & 0.899 & 0.010 & $<0.001$ \\
\hline Age & & 0.003 & 0.001 & $<0.001$ \\
\hline Sex & & 0.001 & 0.004 & 0.710 \\
\hline \multicolumn{5}{|l|}{ Bedtime } \\
\hline$<10: 00$ p.m. & 1275 & - & & - \\
\hline $\begin{array}{l}\text { 10:00 p.m. }<= \\
<11: 00 \text { p.m. }\end{array}$ & 1211 & -0.006 & 0.004 & 0.129 \\
\hline $\begin{array}{l}\text { 11:00 p.m. }<=<0: 00 \\
\text { p.m. }\end{array}$ & 807 & -0.021 & 0.006 & $<0.001$ \\
\hline
\end{tabular}




\begin{tabular}{lclll}
\hline & $N$ & Coefficient & Standard error & $P$ value \\
\hline $\begin{array}{l}\text { 0:00 p.m. }<=<1: 00 \\
\text { a.m. }\end{array}$ & 282 & -0.028 & 0.008 & $<0.001$ \\
1:00 a.m. $<=$ & $59-0.080$ & 0.019 & $<0.001$ \\
\hline
\end{tabular}

\section{Declarations}

Funding The National Institute of Public Health provided funding for the conduct of this study and the preparation of this article.

Conflicts of interest Takeru Shiroiwa and Takashi Fukuda have no conflicts of interest that are directly relevant to the content of this article.

Ethics approval This study was conducted with the approval of the Ethics Committee of the National Institute of Public Health (NIPHIBRA \#12179).

Consent to participate We obtained written informed consent from all the respondents.

Consentfor publication Not applicable.

Data availability Our data are available only to the authors because we obtained informed consent from the respondents under that condition.

Code availability Not applicable.

Author contributions TS and TF were involved in the study design and data interpretation. TS was involved in the statistical analysis. This manuscript was prepared by TS. All the authors participated in revising the report and commenting on the drafts of the manuscript, and approved the final report.

Open Access This article is licensed under a Creative Commons Attribution-NonCommercial 4.0 International License, which permits any non-commercial use, sharing, adaptation, distribution and reproduction in any medium or format, as long as you give appropriate credit to the original author(s) and the source, provide a link to the Creative Commons licence, and indicate if changes were made. The images or other third party material in this article are included in the article's Creative Commons licence, unless indicated otherwise in a credit line to the material. If material is not included in the article's Creative Commons licence and your intended use is not permitted by statutory regulation or exceeds the permitted use, you will need to obtain permission directly from the copyright holder. To view a copy of this licence, visit http://creativecommons.org/licenses/by-nc/4.0/.

\section{References}

1. Stevens K. Developing a descriptive system for a new preference-based measure of health-related quality of life for children. Qual Life Res. 2009;18(8):1105-13. https://doi.org/10.1007/ s11136-009-9524-9.

2. Wille N, Badia X, Bonsel G, Burström K, Cavrini G, Devlin N, et al. Development of the EQ-5D-Y: a child-friendly version of the EQ-5D. Qual Life Res. 2010;19(6):875-86. https://doi.org/10. 1007/s11136-010-9648-y.
3. Feeny D, Furlong W, Torrance GW, Goldsmith $\mathrm{CH}, \mathrm{Zhu} \mathrm{Z}$, DePauw S, et al. Multiattribute and single-attribute utility functions for the health utilities index mark 3 system. Med Care. 2002;40(2):113-28. https://doi.org/10.1097/00005650-20020 2000-00006.

4. Apajasalo M, Sintonen H, Holmberg C, Sinkkonen J, Aalberg $\mathrm{V}$, Pihko $\mathrm{H}$, et al. Quality of life in early adolescence: a sixteen-dimensional health-related measure (16D). Qual Life Res. 1996;5(2):205-11. https://doi.org/10.1007/bf00434742.

5. Apajasalo M, Rautonen J, Holmberg C, Sinkkonen J, Aalberg V, Pihko H, et al. Quality of life in pre-adolescence: a 17-dimensional health-related measure (17D). Qual Life Res. 1996;5(6):532-8. https://doi.org/10.1007/bf00439227.

6. Moodie M, Richardson J, Rankin B, Iezzi A, Sinha K. Predicting time trade-off health state valuations of adolescents in four Pacific countries using the Assessment of Quality-of-Life (AQoL-6D) instrument. Value Health. 2010;13(8):1014-27. https://doi.org/10.1111/j.1524-4733.2010.00780.x.

7. Shiroiwa T, Ikeda S, Noto S, Fukuda T, Stolk E. Valuation survey of EQ-5D-Y based on the international common protocol: development of a value set in Japan. Med Decis Mak. 2021. https://doi.org/10.1007/s11136-015-1108-2.

8. Prevolnik Rupel V, Ogorevc M. EQ-5D-Y value set for Slovenia. Pharmacoeconomics. 2021. https://doi.org/10.1007/ s40273-020-00994-4.

9. Ramos-Goñi JM, Oppe M, Stolk E, Shah K, Kreimeier S, Rivero-Arias $\mathrm{O}$, et al. International valuation protocol for the EQ5D-Y-3L. Pharmacoeconomics. 2020;38(7):653-63. https://doi. org/10.1007/s40273-020-00909-3.

10. Szende A, Janssen B, Cabases J, editors. Self-reported population health: an international perspective based on EQ-5D. Dordrecht: Springer; 2014.

11. Kim TH, Jo MW, Lee SI, Kim SH, Chung SM. Psychometric properties of the EQ-5D-5L in the general population of South Korea. Qual Life Res. 2013;2(8):2245-53. https://doi.org/10. 1007/s11136-012-0331-3.

12. Hinz A, Kohlmann T, Stöbel-Richter Y, Zenger M, Brähler E. The quality of life questionnaire EQ-5D-5L: psychometric properties and normative values for the general German population. Qual Life Res. 2014;23(2):443-7. https://doi.org/10.1007/ s11136-013-0498-2.

13. McCaffrey N, Kaambwa B, Currow DC, Ratcliffe J. Healthrelated quality of life measured using the EQ-5D-5L: South Australian population norms. Health Qual Life Outcomes. 2016;14(1):133. https://doi.org/10.1186/s12955-016-0537-0.

14. Garcia-Gordillo MA, Adsuar JC, Olivares PR. Normative values of EQ-5D-5L: in a Spanish representative population sample from Spanish Health Survey, 2011. Qual Life Res. 2016;25(5):1313-21. https://doi.org/10.1007/ s11136-015-1164-7.

15. Golicki D, Niewada M. EQ-5D-5L Polish population norms. Arch Med Sci. 2017;13(1):191-200. https://doi.org/10.5114/aoms. 2015.52126.

16. Yang Z, Busschbach J, Liu G, Luo N. EQ-5D-5L norms for the urban Chinese population in China. Health Qual Life Outcomes. 2018;16(1):210. https://doi.org/10.1186/s12955-018-1036-2.

17. Purba FD, Hunfeld JAM, Iskandarsyah A, Fitriana TS, Sadarjoen SS, Passchier J, et al. Quality of life of the Indonesian general population: test-retest reliability and population norms of the EQ5D-5L and WHOQOL-BREF. PLoS One. 2018;13(5): e0197098. https://doi.org/10.1371/journal.pone.0197098.

18. Hernandez G, Garin O, Pardo Y, Vilagut G, Pont À, Suárez M, et al. Validity of the EQ-5D-5L and reference norms for the Spanish population. Qual Life Res. 2018;27(9):2337-8. https://doi.org/ 10.1007/s11136-018-1877-5. 
19. Hobbins A, Barry L, Kelleher D, O'Neill C. The health of the residents of Ireland: Population norms for Ireland based on the EQ-5D-5L descriptive system: a cross sectional study. HRB Open Res. 2018;1:22. https://doi.org/10.12688/hrbopenres.12848.1.

20. Bailey H, Janssen MF, La Foucade A, Kind P. EQ-5D-5L population norms and health inequalities for Trinidad and Tobago. PLoS One. 2019;14(4):e0214283. https://doi.org/10.1371/journ al.pone.0214283.

21. Grochtdreis T, Dams J, König HH, Konnopka A. Health-related quality of life measured with the EQ-5D-5L: estimation of normative index values based on a representative German population sample and value set. Eur J Health Econ. 2019;20(6):933-44. https://doi.org/10.1007/s10198-019-01054-1.

22. Wong EL, Cheung AW, Wong AY, Xu RH, Ramos-Goñi JM, Rivero-Arias $\mathrm{O}$. Normative profile of health-related quality of life for Hong Kong general population using preference-based instrument EQ-5D-5L. Value Health. 2018;22(8):916-24. https://doi.org/10. 1016/j.jval.2019.02.014.

23. Prevolnik Rupel V, Ogorevc M. EQ-5D-5L Slovenian population norms. Health Qual Life Outcomes. 2020;18(1):333. https://doi. org/10.1186/s12955-020-01584-w.

24. Poder TG, Carrier N, Kouakou CRC. Quebec health-related quality-of-life population norms using the EQ-5D-5L: decomposition by sociodemographic data and health problems. Value Health. 2020;23(2):251-9. https://doi.org/10.1016/j.jval.2019.08.008.

25. Encheva M, Djambazov S, Vekov T, Golicki D. EQ-5D-5L Bulgarian population norms. Eur J Health Econ. 2020;21(8):1169-78. https://doi.org/10.1007/s10198-020-01225-5.

26. Jiang R, Janssen MFB, Pickard AS. US population norms for the EQ-5D-5L and comparison of norms from face-to-face and online samples. Qual Life Res. 2021;30(3):803-16. https://doi.org/10. 1007/s11136-020-02650-y.

27. Shiroiwa T, Fukuda T, Ikeda S, Igarashi A, Noto S, Saito $\mathrm{S}$, et al. Japanese population norms for preference-based measures: EQ-5D-3L, EQ-5D-5L, and SF-6D. Qual Life Res. 2016;5(3):707-19. https://doi.org/10.1007/s11136-015-1108-2.

28. Shiroiwa T, Fukuda T, Noto S. Japanese population norms of EQ5D-5L and HUI 3: disutility catalogue associated with disease and symptoms in community settings. Value Health. 2021. https://doi. org/10.1016/j.jval.2021.03.010.

29. Herdman M, Gudex C, Lloyd A, Janssen M, Kind P, Parkin D, et al. Development and preliminary testing of the new five-level version of EQ-5D (EQ-5D-5L). Qual Life Res. 2011;20(10):172736. https://doi.org/10.1007/s11136-011-9903-x.

30. Kreimeier S, Oppe M, Ramos-Goñi JM, Cole A, Devlin N, Herdman M, et al. Valuation of EuroQol Five-Dimensional Questionnaire, Youth Version (EQ-5D-Y) and EuroQol Five-Dimensional Questionnaire, Three-Level Version (EQ-5D-3L) health states: the impact of wording and perspective. Value Health. 2018;21(11):1291-8. https://doi.org/10.1016/j.jval.2018.05.002.

31. Euroqol Group. EQ-5D-Y user guide. 2020. https://euroqol.org/ wp-content/uploads/2020/09/EQ-5D-Y-User-Guide_version-2.0. pdf. Accessed 18 Dec 2020.

32. Tsuchiya A, Ikeda S, Ikegami N, Nishimura S, Sakai I, Fukuda $\mathrm{T}$, et al. Estimating an EQ-5D population value set: the case of Japan. Health Econ. 2002;11(4):341-53. https://doi.org/10.1002/ hec.673.

33. Shiroiwa T, Ikeda S, Noto S, Igarashi A, Fukuda T, Saito S, et al. Comparison of value set based on DCE and/or TTO data: scoring for EQ-5D-5L health states in Japan. Value Health. 2016;19(5):648-54. https://doi.org/10.1016/j.jval.2016.03.1834.

34. Henry EB, Barry LE, Hobbins AP, McClure NS, O'Neill C. Estimation of an instrument-defined minimally important difference in EQ-5D-5L index scores based on scoring algorithms derived using the EQ-VT Version 2 valuation protocols. Value Health. 2020;3(7):936-44. https://doi.org/10.1016/j.jval.2020.03.003. 